\title{
Desafíos y propuestas para los ambientes de enseñanza-aprendizaje
}

\author{
Bangesy Carrasquillo-Casado. Universidad Metropolitana (Puerto Rico - Estados Unidos) \\ Recepción: 18 de abril de 2016 | Revisión: 4 de mayo de 2016 | Aceptado: 9 mayo de 2016 \\ Correspondencia: bcarrasquillo18@suagm.edu \\ Citar: Carrasquillo-Casado, B. (2016). Desafíos y propuestas para los ambientes de enseñanza-aprendizaje. ReiDoCrea, 5, 97-102.
}

\begin{abstract}
Resumen: Este artículo tiene como objetivo reseñar el libro: La escuela y los ambientes y contextos de la enseñanzaaprendizaje. Es una compilación de cinco artículos que profundizan en diversos aspectos de la educación escolar y el estado de situación actual según los renglones económicos, sociales, culturales, científicos y tecnológicos dentro del contexto de la sociedad del conocimiento. Este libro es parte de la Serie: Desafíos de la Educación en un Mundo Complejo de Universitas Nueva Civilización en Chile, resultado de un ciclo de investigación de varios colaboradores en temas relacionados con la diversidad, pobreza, igualdad de oportunidades de género, integración y justicia social, formación de educadores, desescolarización, entre otros. En cada texto, sus autores y autoras presentan propuestas prácticas a los problemas socio-educativos desde una perspectiva y enfoque críticos. Nuestro propósito es resaltar la educación democrática y las comunidades de aprendizaje como alternativas para la transformación social y educativa.
\end{abstract}

Palabras clave: Educación | Ambiente Educacional

Challenges and new approaches for teaching and learning environments

\begin{abstract}
This article is to review the book: La escuela y los ambientes y contextos de la enseñanza-aprendizaje, a compilation of five articles that delve into various aspects of school education and the status of the current situation according to economic, social, cultural, scientific and technological aspects within the context of the knowledge society. This book is part of the series: Desafíos de la Educación en un Mundo Complejo of Universitas Nueva Civilización in Chile. Also is a result of a cycle of research about diversity, poverty, equal gender opportunities, integration and social justice, teacher's professional development, unschooling, among others. In each text, its authors present a critical approach and practical proposals to socio-educational issues. Our purpose is to highlight the democratic education and learning communities as new alternative ways to reach a social and educational transformation.
\end{abstract}

Keywords: Education | Learning environment

\section{Introducción}

Estudiar el modelo de las comunidades de aprendizaje nos vincula a una serie de textos que son diversos y multitemáticos tanto como lo es una comunidad en sí misma. Precisamente, como parte de la revisión de literatura que realizo en la investigación acerca de las comunidades de aprendizaje en la educación democrática: estudio de caso en Puerto Rico, encontré el libro La escuela y los ambientes y contextos de la enseñanzaaprendizaje (Arnt, R., Canihuante, G., Moreno, B., Pereira, P., Razeto, L., Razeto, P., Richards, H., Valdebenito, A. y Valenzuela, C., 2014). Con la visita de uno de los autores principales, el Dr. Luis Razeto Migliaro a Puerto Rico en diciembre 2015, y luego de presenciar las conferencias y conversaciones con diversos sectores sociales acerca de la economía de solidaridad me propuse reseñar este texto que vincula la educación como eje central del desarrollo de un movimiento de país. Actualmente, el archipiélago puertorriqueño atraviesa uno de los periodos económicos más críticos de la historia desde la llamada Gran Depresión de 1930. Y, acompañada a la crisis, el sistema de educación pública sufre cambios radicales que pretenden ser respuesta y solución a los problemas. Algunas de las iniciativas van desde el desarrollo de comunidades profesionales de aprendizaje, la integración de métodos alternativos como la filosofía Montessori a la escuela tradicional pública, la consolidación de grados, revisiones curriculares e integración de materias hasta la implantación de pruebas estandarizadas como criterio de evaluación sumativa del aprendizaje estudiantil. A la situación se le añade el cierre de 
decenas de planteles escolares debido a la disminución en la matrícula estudiantil generada por los fracasos escolares, bajas tasas de retención escolar y aumento en la emigración de las familias mayormente hacia Estados Unidos. Por otro lado, la educación privada continúa con la implementación de modelos educativos que abarcan desde lo tradicional-conservador hasta lo alternativo-liberal. Por esta razón es pertinente reseñar este libro en ánimos de explorar soluciones que ubican la educación en su definición amplia ante una nueva mirada. Este texto es una compilación de cinco artículos que profundizan acerca de la situación actual de la educación escolar chilena, afectada por los renglones económicos, sociales, culturales, científicos y tecnológicos dentro del contexto de la sociedad del conocimiento. Cabe resaltar que el mismo es parte de la Serie: Desafíos de la Educación en un Mundo Complejo de Universitas Nueva Civilización en Chile; resultado de un ciclo de investigación de varios colaboradores en temas relacionados con la diversidad, pobreza, igualdad de oportunidades de género, integración y justicia social, formación de educadores, desescolarización, entre otros.

Al inicio del libro, se presenta ante la consideración del lector un diagnóstico crítico de la educación escolar y sus causas principales, que bien pueden generalizarse a regiones en gran parte del mundo. Entre las razones destacan la ausencia de pertinencia de los contenidos a las necesidades de los estudiantes y de la sociedad contemporánea, el uso de métodos tradicionales y poco participativo de enseñanza, la falta de adaptación de las tecnologías de información y comunicación en el currículo. También, menciona el traspaso de conocimientos obsoletos por parte del maestro a los estudiantes debido a la ausencia de formación profesional de calidad, así como las desigualdades sociales y económicas entre los miembros de la comunidad escolar. Resaltan la estandarización e institucionalización en los procesos administrativos, de enseñanza y de la evaluación del aprendizaje estudiantil como problemas de la situación educativa actual que descarta la flexibilidad de procesos, admite el desconocimiento o ceguera ante las dimensiones emocionales e ignora el desarrollo integral del ser humano, entre otros. En cada uno de los textos, sus autores y autoras presentan propuestas prácticas a los problemas socioeducativos desde una perspectiva y enfoque críticos.

\section{Discusión}

El primero de los artículos se titula Pluralismo y diversidad en la educación para la integración social y el desarrollo humano escrito por el Dr. Luis Razeto, el Dr. Howard Richards, el Prof. Gustavo Canihuante y el Dr. Pablo Razeto en el que establecen la necesidad de definir los objetivos que permitan la transformación educativa en Chile. Proponen dos objetivos o metas que debe asumir toda actividad o movimiento que busque generar cambios verdaderos. Estos son "la integración social, que implica reducir sustancialmente la enorme desigualdad de condiciones y oportunidades que se manifiesta y reproduce en la educación; y el desarrollo humano y económico-social sustentable que corresponde el ingreso activo de la nación" (Razeto, Richards, Canihuante, \& Razeto Barry, 2014, p. 9). Para el logro de estos objetivos, los autores examinan el fenómeno de la fragmentación social según los sectores socio-económicos desde los vulnerables a los privilegiados, las tecnologías de información y comunicación en la escuela, así como el rol del Estado y de la sociedad civil en la educación. Una exhaustiva mirada a los diversos grupos sociales, estratificado en tres países distintos a los que llaman Chile A (clase alta), Chile BC (clase media) y Chile D (clase baja), la cual busca enfrentar la realidad desigual 
que el sistema escolar ofrece a los participantes según su origen y condición. Sin embargo, a pesar de que resaltan la educación como reproductora de las desigualdades generadas por el sistema económico y político, la proponen como herramienta para erradicar la inequidad (Razeto, et al.). Los autores exploran las prácticas, métodos de enseñanza y materias necesarias para una transformación de país. Sugieren una educación diferenciada que atienda las necesidades de cada sector en la sociedad, donde se observe una vinculación clara de los intereses del individuo y del país. Por otro lado, plantean una filosofía humanista centrada en el valor de la solidaridad y aprendizaje activo, procesos de modernización tecnológica para invertir el tiempo en asuntos de prioridad y procurar referentes de calidad autónomos. Finalmente, discuten la responsabilidad participativa del sector estatal-público, privado y la sociedad civil como factor clave que permita mantener los recursos necesarios para el logro de la calidad en la educación como medio de reinversión social.

En el segundo artículo titulado Educar en los sectores de escasos recursos por y para una nueva civilización el Dr. Howard Richards y el Prof. Andrés Valdebenito, en colaboración con la Profa. Claudia Arcos plantean no estar interesados en preservar el estado de situación actual o promover reformas puramente utilitarias, sino promover la educación como herramienta poderosa que junto a nuevas formas de economía liberal y de solidaridad se convierta en parte integral de la construcción de una nueva civilización que supere la desigualdad. Los autores presentan y discuten varios casos para ejemplificar escenarios de la escuela en Chile según las experiencias y modos de vida heterogéneos de los estudiantes. Entre los casos, resalta a la estudiante que por el alto poder adquisitivo de su familia trata a los maestros como empleados a su servicio, la joven delincuente a la edad de 12 años, la hija de un ingeniero que ha estado expuesta desde temprana edad a juegos matemáticos y pierde el interés de la clase tradicional por encontrar muy sencillo el contenido. Por último, un estudiante que tiene bajas calificaciones y problemas de comprensión debido a una condición de sordera no diagnosticada. Estos ejemplos buscan establecer que la propuesta de "educación igual para todos no sirve para generar equidad porque las desigualdades no se generan en la educación, sino fundamentalmente en el sistema económico y político imperante" (Richards y Valdebenito, 2014, p. 15), sino que la alternativa está en diferenciar la educación según las necesidades. Sugieren sobrepasar los vicios actuales de las reformas del sistema educativo provocadas por la inestabilidad de las políticas gubernamentales y de los intereses de la economía de capital. Es importante resaltar que Richards y Valdebenito (2014) desarrollan sus críticas y propuestas desde una perspectiva académica bajo el análisis de la imposición del clima escolar a la cultura ciudadana. Establecen la necesidad de una educación que apele, modele y promueva valores de solidaridad, autonomía y creatividad como finalidad, mientras que el dinero sea un medio y no un fin. Los autores reconocen que las buenas prácticas de ciertos grupos aportan, pero advierten que no dan paso a una nueva civilización. Por esta razón, se cuestionan la necesidad de fusionar la escuela con la comunidad, las familias y los comercios vecinos para ser "agente catalítico en el saneamiento de la cultura juvenil" (p.65) y hacer la escuela más atractiva a los alumnos con el fin del bienestar humano.

En el tercer artículo, la psicóloga organizacional y profesora Camila Valenzuela Persico escribe acerca de El aprendizaje permanente, la igualdad de oportunidades de género y la justicia social. Inicia enfatizando la necesidad del aprendizaje en el ser humano y la importancia de fomentarlo como una actividad a lo largo de la vida en mujeres y hombres. La autora discute elementos históricos y teóricos del concepto aprendizaje permanente, así como realiza un análisis de justicia social con un enfoque de género (Valenzuela 
Persico, 2014). Se observa la educación como un proceso continuo que no debe discriminar entre métodos formales e informales, que las experiencias sean valoradas y se posea una perspectiva más amplia y humanista. De ahí, que el concepto de justicia social en este artículo apela también al derecho de una educación en las dimensiones personales y sociales además de las laborales o profesionales. En una sociedad en la que la educación está presa de una economía competitiva, el proceso de aprendizaje no debe ceder ciertos principios del estudiante como centro, igualdad de oportunidades, calidad y relevancia. Valenzuela (2014) apunta a la ideología de educación en comunidad, a una pedagogía práctica, activa y experiencial, basada en la teoría del generativismo. La profesora Valenzuela cita a Carneiro (2011) para explicar el generativismo como la búsqueda constante de significado y el derecho pleno a la reconstrucción del conocimiento que va desde la información, el conocimiento, el aprendizaje hasta el desafío de crear nuevo conocimiento desde el previamente codificado. El aprendizaje permanente promueve la autonomía y la innovación desde una perspectiva constructivista para que el aprendiz pueda fortalecer o desarrollar el pensamiento crítico, su capacidad creativa, las relaciones interpersonales, el reconocimiento de la diversidad y la resolución de problemas. Estas destrezas serán necesarias para que cualquier individuo, sin importar su género, supere las desigualdades impuestas, salga de los roles tradicionales y acceda a las libertades reales que corresponden al logro de la justicia social. Una vez más, se presentan ambientes de aprendizaje participativos, transversales a la vida en comunidad, como propuesta de democratizar la educación para el disfrute de un aprendizaje integral en igualdad de oportunidades (Valenzuela, 2014).

En el cuarto artículo, las doctoras Rosamaría de Medeiros Arnt y Paula Pereira presentan en el artículo Principios transdisciplinarios del Programa Generación de Paz: puntos de partida para la extensión, investigación y formación de educadores en el que describen el programa de Extensión de la Universidad Estadual de Ceará en Brasil. Este artículo nos permite reflexionar acerca de los logros y retos de la investigación y formación de educadores para la ciudadanía planetaria y para la paz en entornos comunitarios y escuelas públicas. Las autoras establecen que los principios y métodos utilizados en el Programa de Generación de Paz (PGP) no pretenden ser una guía, sino un grupo de experiencias que han ido formando su identidad con cada proyecto desarrollado. Sin embargo, Arnt de Medeiros y Pereira logran presentar seis principios dinámicos que "deberán ser comprendidos a medida que los individuos y las instituciones interactúen" (2014, p.121) en cualquier práctica educativa. Estos puntos de partida para la generación de paz son: apertura (para la comprensión de la realidad), escucha (atenta y sensible), diálogo (para el surgimiento de nuevas ideas y relaciones), flexibilidad (para aceptar propuestas diferentes, situaciones inesperadas e identificar nuevas alternativas), compartir (participación y contribución) y auto-organización (para el surgimiento de un nuevo orden de las experiencias y necesidades). Con un tono esperanzador a la luz de la concienciación y sensibilización de los educadores, las doctoras Arnt de Medeiros y Pereira cierran su escrito proponiendo la interrogante ¿cuál es el perfil profesional, qué competencias, conocimientos y habilidades deberá tener, y cómo se podrá formar el profesor del futuro?

En el quinto y último de los artículos del texto, el licenciado en Educación y especialista en Biología del Conocer, el Prof. Bastián Moreno, cierra magistralmente con la propuesta Desescolarización de la escuela: hacia el rescate de lo educativo en la que inicia relacionando los conceptos crisis y educación. Plantea la diferencia entre Educación como el "proceso permanente y espontáneo de posibles relaciones de transformación en convivencia" (Moreno, 2014, p. 150) y la Escolarización como un proceso que reproduce 
relaciones predeterminadas. Advierte que la educación ha sufrido un tipo de dominio o apropiación por parte de la escuela, la que con su rigidez y linealidad extingue la naturalidad del proceso educativo lo cual provoca el alejamiento de los estudiantes. Moreno (2014) establece que la sociedad actual exige un cambio de paradigma que no debe ser delegado a las autoridades académicas o científicas, sino que las reformulaciones deben darse desde las relaciones entre los miembros de las comunidades docentes y no docentes. Luego de criticar los postulados del paradigma positivista tradicional y el enfoque del contenido comparado con el énfasis del paradigma emergente de aprender a aprender, el autor asegura que éste último goza de sistemas igualitarios de participación, creatividad, indicadores cualitativos de "conocimiento para el vivir" (p.162) y promueve la coexistencia entre aprendices y educadores en responsabilidad compartida. La propuesta del profesor Bastián Moreno de desescolarización busca el aprendizaje mediado por la convivencia y no por el contenido. Asimismo, entiende que es necesario recuperar el rol educativo de la escuela como medio social natural a través del reconocimiento del educando y los educadores como responsables de construir relaciones (formales e informales) de aceptación y respeto a sí mismos y al proceso de enseñanza y aprendizaje.

\section{Conclusiones}

No cabe duda que la educación como proceso y como sistema es esencial para el desarrollo del ser humano y su sociedad. También es "uno de los ámbitos sociales a los que más le cuesta cambiar" (Díez y Flecha, 2010, p. 19). Sin embargo, las relaciones humanas representan un centro de acción capaz de generar proyectos para la transformación social desde la edificación de entornos de aprendizaje participativos y multicuturales que den paso a la educación de todos. En Puerto Rico, al igual que en otros países el desafío está en la urgencia de una educación que emerja para lograr su función principal de despertar conciencias en un ambiente con diversidad de situaciones, pero con alternativas reales para los miembros de la sociedad. Elise Freinet (1977) repasando la trayectoria de la pedagogía popular de Celestín Freinet, indicaba que cada día él llegaba a la misma conclusión: "la enseñanza tradicional que exige del niño una actitud pasiva y amorfa está condenada al fracaso" (p. 18). Como gestor de una pedagogía popular y líder investigador de un movimiento para la escuela moderna, Freinet (1977) insistía que "la razón de su investigación en el campo de la educación era salvar a los niños del autoritarismo irracional de los adultos y mostrarles nuevas formas de emancipación individual y social" (p. 139). Es el dominio absoluto que existe por parte de los sistemas educativos a través de modelos y estrategias de enseñanza retrógrados lo que provoca la pasividad y resignación social que estamos sufriendo. La infancia y juventud de los sectores desventajados de nuestro país está a merced de técnicas educativas que resultan en precariedad cognitiva y en conductas de inacción contrapuestas a las habilidades que requieren para la vida en general. Además, esta nueva era de conexiones exige armonizar lo individual con lo social y político para la realización futura del individuo ante su sociedad. Según Díez y Flecha (2010) el modelo educativo de las comunidades de aprendizaje es una respuesta eficiente a los cambios sociales y educativos introducidos por la sociedad de la información. Es por esta razón que nuevos espacios educativos en comunidad de aprendizaje donde la conversación, la pregunta y la exploración alineada a los intereses y necesidades de los participantes serán los que han de emerger para atender los problemas existentes en un contexto de rápidos cambios. Según Elboj y Oliver (2003) trabajar en comunidades de aprendizaje como modelo educativo permite colaborar en la superación de las desigualdades 
educativas y ser coherentes con una sociedad que es cada vez más dialógica para dar respuesta a sus conflictos.

Por esta razón, al repasar cada uno de los textos de Arnt, et. al (2014), vemos reflejado el movimiento de una sociedad que exige cambios y que va en busca de vivir una filosofía humanista que evoque principios de justicia social, respeto, paz, honestidad, servicio y solidaridad. Todo ello bajo nuevas visiones de la educación, que a su vez conlleva una responsabilidad de todos y todas en reconocer el placer y la importancia de aprender en condiciones de equidad, salvaguardando la sustentabilidad económica y la salud. Sin embargo, para responder a todas estas necesidades "la escuela no puede actuar sola" (Elboj y Oliver, 2003, p. 96); la mejor forma de conseguir los objetivos es a través de la participación de la comunidad vinculando la economía, la política y la educación como activos sociales. Esto nos permite reflexionar acerca de la necesidad de investigar e implementar nuevas opciones educativas que desarrollen destrezas interpersonales y valores sociales democráticos en infantes y jóvenes para la reorientación hacia una nueva civilización. No será jamás la educación y los educadores los únicos responsables del cambio social, pero una acción educativa mejor organizada, participativa y democrática, centrada en las comunidades y sus necesidades y no solo en los individuos será capaz de aspirar a una verdadera transformación.

\section{Referencias}

Arnt, R., Canihuante, G., Moreno, B., Pereira, P., Razeto, L., Razeto, P., Ricards, H., Valdebenito, A. y Valenzuela, C. (2014). La escuela y los ambientes y contextos de la enseñanza-aprendizaje. Santiago de Chile: Ediciones Universitas Nueva Civilización.

Arnt, R. y Pereira Scherre, P. (2014). Principios transdisciplinarios del Programa Generación de Paz: puntos de partida para la extensión, investigación y formación de educadores. En Universitas Nueva Civilización (Eds.), La escuela y los ambientes y contextos de la enseñanza-aprendizaje Serie: Desafíos de la educación en un mundo complejo (pp.117-148). Santiago de Chile: Ediciones Universitas Nueva Civilización.

Díez-Palomar, J. y Flecha García, R. (2010). Comunidades de aprendizaje: un proyecto de transformación social y educativa. Revista Interuniversitaria de Formación del Profesorado 67(24), 19-30.

Elboj Saso, C. y Oliver Pérez, E. (2003). Las comunidades de aprendizaje: un modelo de educación dialógica en la sociedad del conocimiento. Revista Interuniversitaria de Formación del Profesorado 17(3), 91-103.

Freinet, E. (1977). Nacimiento de una pedagogía popular, Historia de una Escuela Moderna (2ª. ed.). Barcelona, España: Editorial Laia.

Moreno, B. (2014). Desescolarización de la escuela: hacia el rescate de lo educativo. En Universitas Nueva Civilización (Eds.), La escuela y los ambientes y contextos de la enseñanza-aprendizaje Serie: Desafíos de la educación en un mundo complejo (pp. 149-184). Santiago de Chile: Ediciones Universitas Nueva Civilización.

Razeto, L., Richards, H., Canihuante, G. y Razeto Barry, P. (2014). Pluralismo y diversidad en la educación para la integración social y el desarrollo humano. En Universitas Nueva Civilización (Eds.), La escuela y los ambientes y contextos de la enseñanza-aprendizaje Serie: Desafíos de la educación en un mundo complejo (pp. 7- 49). Santiago de Chile: Ediciones Universitas Nueva Civilización.

Richards, H. y Valdebenito, A. (2014). Educar en los sectores de escasos recursos por y para una nueva civilización. En Universitas Nueva Civilización (Eds.), La escuela y los ambientes y contextos de la enseñanza-aprendizaje Serie: Desafíos de la educación en un mundo complejo (pp. 51-78). Santiago de Chile: Ediciones Universitas Nueva Civilización.

Valenzuela Persico, C. (2014). El aprendizaje permanente, la igualdad de oportunidades de género y la justicia social. En Universitas Nueva Civilización (Eds.), La escuela y los ambientes y contextos de la enseñanza-aprendizaje Serie: Desafíos de la educación en un mundo complejo (pp. 79-116). Santiago de Chile: Ediciones Universitas Nueva Civilización. 